\title{
Avaliação in vitro de uma cepa de campo de Boophilus microplus (Acari : Ixodidae) resistente à Amitraz
}

\author{
In vitro evaluation of a field strain of Boophilus microplus \\ (Acari:ixodidae) resistant to Amitraz
}

\author{
Minerva Santamaría Vargas ${ }^{1}$ Noé Soberanes Céspedes ${ }^{1}$ \\ Hugo Fragoso Sánchez ${ }^{1}$ João Ricardo Martins ${ }^{2}$ \\ Carlos Octávio Cordovés Céspedes. ${ }^{3}$
}

\section{RESUM0}

O uso dos acaricidas químicos ainda se constitui no principal instrumento de controle do carrapato bovino Boophilus microplus. No sul do Brasil, o amitraz aplicado por imersão e aspersão é o ingrediente ativo mais utilizado, nos últimos anos, contra as cepas de carrapatos resistentes aos organofosforados (OF) e piretróides sintéticos (PS). Em conseqüencia, torna-se importante a realização de investigações que possam contribuir para prolongar a vida útil desse ixodicida. No presente estudo, foi analisado o comportamento toxicológico de uma cepa de B. microplus colhida na localidade de Alegrete, Rio Grande do Sul, usandose químicos da familia dos $O F, P S$, misturas $O F / P S$, além do amitraz. Os resultados indicaram que a cepa denominada "Santa Luiza" apresentou fatores de resistência (FR) que variaram entre 2,3 e 3,95 para $O F, 23,3$ e 147,56 para $P S$ e de 3,76 a 21.57 para amitraz em testes realizados com larvas de carrapatos. A caracterização e purificação de uma cepa de B. microplus resistente ao amitraz permitem seu uso como cepa de referência para a avaliação biológica de ixodicidas alternativos, além de padronizar e validar métodos de diagnósticos toxicológicos que possam detectar resistência frente a esse acaricida.

Palavras-chave: amitraz, carrapato, Boophilus microplus, resistência.

\section{ABSTRACT}

The use of chemical acaricide still remains as the main tool against the cattle tick Boophilus microplus. In Southern Brazil, amitraz applied by immersion and/or spray is the main active ingredient to control organophosphate and synthetic pyrethroid resistant strains. In consequence it is important to conduct investigations in order to prolong useful life of this ingredient. In this article, toxicological behaviour of a strain of Boophilus microplus named "Santa Luiza" collected in the locality of Alegrete, state of Rio Grande do Sul, Brazil, was analyzed against acaricides organophosphorous, pyrethroids, mixtures $S P$ and $O P$ and amitraz. Results showed factors of resistance from 2.3 to 3.95 to OP, 23.3 to 147.56 to SP and 3.76 to 21.57 to amitraz indicating that this strain showed multiple resistance to tested acaricides. Characterization and purification of a $\boldsymbol{B}$. microplus strain resistant to amitraz will allow its use as a reference strain for future utilization in biological evaluation of alternative acaricides as well as to standard and to validate diagnostic methods to detect resistant to this acaricide.

Key words: Amitraz, tick, Boophilus microplus, resistance.

\section{INTRODUÇÃO}

O desenvolvimento de resistência do carrapato Boophilus microplus ao amitraz surgiu após quatro a dez anos de seu uso contínuo em diferentes partes do mundo. Esta situação foi inicialmente evidenciada na Austrália, onde, em 1980, identificouse a cepa denominada "Ulam", a qual apresentou altos níveis de resistência ao amitraz e resistência cruzada ao cymiazol e ao chlorometiuron, com uma limitada distribuição inicial de 14 casos que alcançaram a 30 durante o período de 1990 a 1998 (NOLAN, 1981; KEMP et al., 1998). Ao final de 1992, detectou-se resistência em 40 fazendas, quatro anos após o uso contínuo do

${ }^{1}$ Médico Veterinário, Centro Nacional de Servicios de Constatación en Salud Animal. SENASICA-SAGARPA, Km 11.5, Carr. Fed. Cuernavaca-Cuautla, Col. Progreso Jiutepec, Morelos, México. CP. 62550.

${ }^{2}$ Médico Veterinário, Instituto de Pesquisas Veterinarias "Desiderio Finamor" - FEPAGRO, Estrada do Conde, 6000, 92990-000, Eldorado do Sul-RS, Brasil. E-mail: joaorsm@zaz.com.br Autor para correspondência.

${ }^{3}$ Médico Veterinário, Bolsista CNPq, Produtos Veterinários Ouro Fino, Rua Vicente Golfeto, 59. Ribeirão Preto, São Paulo-SP. 
amitraz, quando foi identificada e caracterizada uma cepa conhecida como "Ultimo", resistente a todos os piretroides e ao amitraz (KUNZ \& KEMP, 1994). Na África do Sul e Colômbia, também registraram-se populações de $\boldsymbol{B}$. microplus resistentes ao amitraz (KUNZ \& KEMP, 1994; KEMP et al., 1998; STRYDOM \& PETER, 1999) enquanto que, no Brasil, relatos de resistência ao amitraz foram notificados em alguns estados (MARTINS et. al., 1995, FURLONG, 1999).

No México, a resistência aos organofosforados e aos piretróides foi diagnosticada em 1993 (ORTIZ et al., 1995), através de bioensaios de laboratório e testes de campo. A partir dessa data, o uso do amitraz foi intensificado, motivo pelo qual se implementaram programas de vigilância no campo a fim de se determinar possíveis alterações na susceptibilidade ao amitraz em populações naturais de $\boldsymbol{B}$. microplus que tenham sido pressionadas com esse produto (SANTAMARIA et al., 1999).

O propósito do presente estudo foi avaliar uma cepa de campo de B. microplus resistente ao amitraz no Brasil, para caracterizar sua resposta toxicológica contra esse e outros carrapaticidas de uso comum no México e no Brasil. Com a informação obtida, pretende-se fornecer subsídios para a implementação de estratégias de manejo que possam retardar o estabelecimento e a dispersão de cepas de carrapatos B. microplus resistentes ao amitraz.

\section{MATERIAL E MÉTODOS}

Os ensaios in vitro e o cultivo das cepas de carrapatos testadas foram realizados no "Centro Nacional de Servicios de Constatación en Salud Animal" (CNSCSA), SENASICA-SAGARPA, Jiutepec, Morelos, México.

A cepa resistente ao amitraz utilizada nesta investigação originou-se de teleóginas de $\boldsymbol{B}$. microplus colhidas em bovinos no município de Alegrete, RS, Brasil. A mesma foi denominada "Santa Luiza", local onde se constataram falhas de controle com o uso de acaricidas organofosforados, piretróides e amitraz. A segunda cepa de carrapatos B. microplus, denominada "suscetível", foi utilizada como cepa de referência e foi obtida no estado de Guerrero, México, em 1997, local sem histórico de uso de carrapaticidas.

Os acaricidas usados, nos testes in vitro, foram os organosfosforados: coumafós, clorfenvinfos e diazinon; os piretróides: flumetrina, deltametrina e cipermetrina, em grau técnico para a Técnica do Pacote de Larvas (LPT) (STONE \& HAYDOCK, 1962). Os carrapaticidas na formulação de concentrado em emulsão comercializados no México e que foram utilizados para a Técnica de Imersão de Adultos (AIT) (DRUMMOND et al., 1976) foram o Taktic ${ }^{\circledR}$ (amitraz $12,5 \%$ de ingrediente ativo, ia), Bovithion ${ }^{\circledR}$ (Ethión $68 \%$ ia), Dursban ${ }^{\circledR}$ (clorpirifós 24\% ia), Asuntol ${ }^{\circledR}$ (coumafós $20 \%$ ia), Supona ${ }^{\circledR}$ (clorfenvinfós $20 \%$ ia), Bayticol ${ }^{\circledR}$ (flumetrina 3\% ia), Butox ${ }^{\circledR}$ (deltametrina $2,5 \%$ ia), Batestan ${ }^{\circledR}$ (cipermetrina $20 \%$ ia), Ectogan ${ }^{\circledR}$ (cymiazol 17,5\% + cipermetrina high-cis 2,5\% ia) y Garraban ${ }^{\circledR}$ (permetrina 5\% + clorpirifos $24 \%$ ia) na concentração comercial recomendada. Para a Técnica de Imersão Larval (LIT) (SHAW, 1966) utilizou-se o Taktic ${ }^{\circledR}(12,5 \%$ ia). Esses acaricidas foram selecionados porque são os registrados pela Direção Geral de Saúde Animal do México, e, atualmente, são os utilizados para o controle dos carrapatos $\boldsymbol{B}$. microplus nesse país. A menção no presente artigo de produtos ixodicidas comerciais não constitui um compromisso ou recomendação para seu uso pelo "Centro Nacional de Servicios de Constatación en Salud Animal”, Cuernavaca, México, nem pelo Instituto de Pesquisas Veterinárias Desidério FinamorFEPAGRO, Eldorado do Sul, RS.

Para os bioensaios, 20.000 larvas da cepa "Santa Luiza" da geração $\mathrm{F}_{5}$ foram colocadas sobre bovinos estabulados para produção de teleóginas. Aos 21 dias, parte das mesmas foi colocada em placas de Petri descartáveis (10 fêmeas/placa) e mantida em incubação a $28^{\circ} \pm 2{ }^{\circ} \mathrm{C}$ e $80-90 \%$ de umidade relativa (UR). A oviposição foi retirada aos 14 dias após a incubação e mensurada, colocando-se um grama de ovos em frascos de vidro, tamponados com algodão, e incubados até a eclosão larval. Para realizar a pressão de seleção com o amitraz, utilizaram-se aproximadamente, 20.000 larvas (1 $g$ de ovos), que foram colocadas em pacotes de papel filtro, com duas repetições. As larvas da geração $F_{6}$ foram tratadas por imersão durante 10 minutos com amitraz na concentração de $0,00004 \%$. Após a exposição ao amitraz, as larvas foram incubadas durante $72 \mathrm{~h}$. As larvas sobreviventes foram utilizadas na infestação de um outro bovino para obtenção da $\mathrm{F}_{7}$.

Os bioensaios, para estabelecer as doses discriminatórias $\left(2 \times \mathrm{CL}_{99}\right)$, foram obtidos com as larvas da geração $F_{1}$ da cepa "Santa Luiza" e a geração $F_{7}$ da cepa "suscetível", com os seguintes acaricidas em grau técnico: coumafos $(0,2 \%)$, clorfenvinfos $(0,2 \%)$, diazinon $(0,08 \%)$, flumetrina $(0,01 \%)$, deltametrina $(0,09 \%)$ e cipermetrina $(0,5 \%)$. Para o cálculo da concentração letal $50\left(\mathrm{CL}_{50}\right)$ dos organofosforados e piretróides e estimativa do fator de resistência (FR) da cepa "Santa Luiza", usou-se o LPT, com seis concentrações e duas repetições, submetendo-se os dados a análise Probit (ROBERTSON \& PREISLER, 1992). 
A Técnica de Imersão de Adultos foi utilizada para avaliação dos percentuais de controle frente aos carrapaticidas comercialmente formulados. Para estes testes, utilizou-se, nas infestações, larvas com trinta dias de idade da geração $F_{1}$ da cepa "Santa Luiza" e da geração $F_{7}$ da cepa "suscetível". Em placas de Petri com $9 \mathrm{~cm}$ de diâmetro, foram colocadas 10 teleóginas de B. microplus com um peso homogêneo de $250 \pm 20 \mathrm{mg}$, formando-se dois grupos, um tratado e um controle, com quatro repetições cada um. As teleóginas dos diferentes grupos tratados foram submersas, durante um minuto, no carrapaticida formulado e os grupos controles foram submersos em água por igual período. Com os dados do peso da massa de ovos e o percentual de eclosão larval, calculou-se o índice de reprodução (DRUMMOND et al., 1976), o qual foi utilizado para a determinação do percentual de controle com o amitraz, organofosforados, piretróides e as misturas OF/PS.

Para se determinar a suscetibilidade ao amitraz, utilizou-se a Técnica de Imersão Larval, utilizando-se três repetições da dose discriminante de $0,0002 \%$, com número igual de repetições para o grupo controle. Mediante a mesma técnica e utilizando-se doze concentrações com três repetições, calculou-se a CL da cepa "susceptível" e da "Santa Luiza" para a determinação do FR, mediante a análise Probit (ROBERTSON \& PREISLER, 1992).

Nos bioensaios in vitro, mediante a técnica do pacote de larvas para organofosforados, piretróides e amitraz, os dados obtidos foram submetidos à análise Probit utilizando-se o programa POLO-PC (LE ORA SOFTWARE, 1987) para se estabelecer o valor da $\mathrm{CL}_{50}$, intervalo de confiança a $95 \%$ e a inclinação ("slope") da linha de regressão. Os valores dos FR foram calculados dividindo-se a $\mathrm{CL}_{50}$ da cepa "Santa Luiza" pela $\mathrm{CL}_{50}$ da cepa "suscetível" (ROBERTSON \& PREISLER, 1992).

\section{RESULTADOS}

Os resultados obtidos no tratamento de larvas com as doses discriminantes realizados com a cepa brasileira "Santa Luiza" indicaram resistência aos organofosforados com percentuais de mortalidade de 100, 96, 95 e 88,35\% para coumafós, clorfenvinfós e diazinon, respectivamente. No caso dos piretróides, a resistência foi maior com percentuais de mortalidade de $9,80 \%$ para flumetrina, $15,16 \%$ para deltametrina e $15,55 \%$ para cipermetrina (Tabela 1). Esses resultados confirmaram resistência a estas duas famílias de ixodicidas.
Tabela 1 - Percentuais de mortalidade de larvas do carrapato bovino B. microplus, cepa "Santa Luiza", colhidas em Alegrete, RS, Brasil, obtidos através da técnica de doses discriminantes (DD).

\begin{tabular}{lcc}
\hline Carrapaticida & $\begin{array}{c}\text { DD* } \\
(\%)\end{array}$ & $\begin{array}{c}\text { Percentual de } \\
\text { mortalidade (\%) }\end{array}$ \\
\hline ORGANOFOSFORADOS $^{\mathrm{a}}$ & & \\
Coumafós & 0,2 & 100,00 \\
Clorfenvinfos & 0,2 & 96,95 \\
Diazinon & 0,08 & 88,35 \\
PIRETROIDES & & \\
Cipermetrina & 0,5 & 15,55 \\
Deltametrina & 0,09 & 15,16 \\
Flumetrina & 0,01 & 9,80 \\
\hline
\end{tabular}

${ }^{\text {a }}$ Carrapaticida em grau técnico

* Dose discriminante $\left(2 \times \mathrm{CL}_{99}\right)$

Através da análise Probit, determinou-se a resposta dose-mortalidade apresentada pela geração $\mathrm{F}_{1}$ da cepa "Santa Luiza" frente aos organofosforados. Registraram-se fatores de resistência de 2,30 para o coumafós, 3,66 para o clorfenvinfós e 3,95 para o diazinon. Três anos após e mantendo-se a cepa sem pressão de seleção com organofosforados, determinou-se novamente a resposta a essa família química mediante testes de dose-mortalidade com a geração $\mathrm{F}_{7}$, obtendo-se para o coumafós, clorfenvinfós e o diazinon, um FR de 2,86, 2,22 e 3,09, respectivamente (Tabela 3). Para o piretróide flumetrina, a cepa "Santa Luiza" na geração F apresentou um FR de 147,56, para a deltametrina um FR de 70,61 e para a cipermetrina, 41,70. Da mesma forma que para os organofosforados, estes bioensaios foram repetidos três anos mais tarde, obtendo-se para a flumetrina, deltametrina e a cipermetrina, um FR de $56,29,39,21$ e de 23,3, respectivamente (Tabela 4).

Com relação aos testes de Imersão de Adultos com a cepa "Santa Luiza", o Besuntol ${ }^{\circledR}$ e o Taktic ${ }^{\circledR}$ mostraram um percentual de 58,69 e $58,41 \%$ de controle, respectivamente, o organofosforado Dursban ${ }^{\circledR}$ atingiu um controle de $99,65 \%$, enquanto que o Supona ${ }^{\circledR}$, Asuntol ${ }^{\circledR}$ e o Bovithion ${ }^{\circledR}$, apresentaram um controle de 69,47, 21,44 e 0,0\%, respectivamente. Os piretróides Bayticol ${ }^{\circledR}$, Butox ${ }^{\circledR} \mathrm{e}$ Batestan $\AA$ apresentaram um controle de 83,69, 26,65 e $1,04 \%$ para cada produto. Com relação às misturas carrapaticidas, o Garraban ${ }^{\circledR}$ obteve um controle de

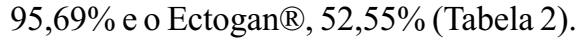

Os resultados obtidos com a Técnica de Imersão Larval, durante a geração $F_{1}$ da cepa "Santa Luiza" apresentaram uma regressão de 1,47 com uma $\mathrm{CL}_{50}$ de $0,00004 \%$ e um $\mathrm{IC}_{95}$ de $0,00002-0,00006$. Nos 
Tabela 2 - Percentuais de controle obtidos no teste de imersão com teleóginas da cepa "Santa Luiza" de B. microplus frente a diferentes carrapaticidas formulados em concentrado emulsionável.

\begin{tabular}{|c|c|c|c|}
\hline FAMILIA Ixodicida & Principio ativo & $\mathrm{CCR}^{*}(\%)$ & Percentual de controle $(\%)$ \\
\hline \multicolumn{4}{|l|}{ AMIDINA } \\
\hline Besuntol** & Cymiazol & 0,03125 & 58,69 \\
\hline Taktic** & Amitraz & 0,025 & 58,41 \\
\hline \multicolumn{4}{|c|}{ ORGANOFOSFORADOS } \\
\hline Dursban** & Clorpirifós & 0,024 & 99,65 \\
\hline Supona** & Clorfenvinfós & 0,030 & 69,47 \\
\hline Asuntol** & Coumafós & 0,020 & 21,44 \\
\hline Bovithion** & Ethion & 0,068 & 0,0 \\
\hline \multicolumn{4}{|l|}{ PIRETRÓIDES } \\
\hline Bayticol** & Flumetrina & 0,030 & 83,69 \\
\hline Butox** & Deltametrina & 0,0025 & 26,65 \\
\hline Batestan** & Cipermetrina & 0,020 & 1,04 \\
\hline \multicolumn{4}{|l|}{ MISTURAS } \\
\hline Garraban & Permetrina + Clorpirifós & 0,29 & 95,69 \\
\hline Ectogan** & Cymiazol+Cipermetrina high cis & 0,030 & 52,55 \\
\hline
\end{tabular}

CCR Concentração comercial recomendada.

** Nome comercial registrado no México.

bioensaios realizados com a geração $\mathrm{F}_{7}$, a cepa "Santa Luiza" apresentou uma regressão de 2,37, uma $\mathrm{CL}_{50} \mathrm{de}$ 0,00023 e um $\mathrm{IC}_{95}$ de 0,00019-0,00026. Em ambos os casos, a cepa "suscetível" obteve uma regressão de 2,24 e uma $\mathrm{CL}_{50}$ de $0,00001 \%$ com um $\mathrm{IC}_{95}$ de 0,00001 0,00002 .

\section{DISCUSSÃO}

De acordo com os resultados da análise Probit, após a manutenção da cepa "Santa Luiza" sem pressão química com carrapaticidas, durante seis gerações, mantiveram-se os FR para os organofosforados coumafós, clorfenvinfós e diazinon. Com relação aos FR obtidos com os piretróides, no caso da flumetrina, houve uma modificação de uma resistência muito alta $(147,56)$ para uma resistência alta $(56,29)$. Em relação à deltametrina, manteve-se o nível de resistência alta $(70,61$ a 39.21) e para a cipermetrina diminuiu de uma resistência alta $(41,70)$ a moderada $(23,3)$ (STONE, 1972). A diminuição observada nos FR obtidos com a cepa "Santa Luiza" para os carrapaticidas piretróides, com uma homozigose de resistência de $60 \%$, mostrou que a manutenção em

Tabela 3 - Determinação das concentrações letais 50 e fatores de resistência da cepa "Santa Luiza" de B. microplus com carrapaticidas organofosforados através do teste do pacote de larvas.

\begin{tabular}{lccccc}
\hline Cepa & Ixodicida* & $\begin{array}{c}\text { Inclinação } \\
\text { (Erro padrão) }\end{array}$ & CL $_{50}^{* *}$ & IC 95\%*** & Fator de resistência \\
\hline "Suscetível" $F_{7}$ & & $7,86(0,58)$ & 0,027 & $0,022-0,034$ & 2,30 \\
"Santa Luiza" $F_{1}$ & Coumafós & $3,85(0,24)$ & 0,063 & $0,050-0,081$ & 2,86 \\
"Santa Luiza" $F_{7}$ & & $9,50(0,80)$ & 0,080 & $0,075-0,084$ & 3,66 \\
"Suscetível" $F_{7}$ & & $7,29(0,55)$ & 0,035 & $0,030-0,039$ & 2,22 \\
"Santa Luiza" $F_{1}$ & Clorfenvinfós & $3,49(0,18)$ & 0,128 & $0,097-0,167$ & 3,95 \\
"Santa Luiza" $F_{7}$ & & $4,85(0,28)$ & 0,078 & $0,067-0,090$ & 3,09 \\
"Suscetível" $F_{7}$ & & $9,01(0,87)$ & 0,012 & $0,010-0,014$ & $0,037-0,057$ \\
"Santa Luiza" $F_{1}$ & Diazinon & $2,05(0,10)$ & 0,045 & $0,030-0,043$ & \\
"Santa Luiza" $F_{7}$ & & $2,73(0,12)$ & 0,036 & & \\
\hline
\end{tabular}

*Grau técnico.

** $\mathrm{CL}_{50}$ Concentração letal 50

$* * * \mathrm{IC}_{95}$ Intervalo de confiança a $95 \%$. 
Tabela 4. Determinação das concentrações letais 50 e fatores de resistência da cepa "Santa Luiza" de B. microplus com carrapaticidas piretróides através do teste do pacote de larvas.

\begin{tabular}{|c|c|c|c|c|c|}
\hline Cepa & Carrapaticida* & $\begin{array}{c}\text { Inclinação } \\
\text { (Erro Padrão) }\end{array}$ & $\mathrm{CL}_{50} * *$ & IC $95 \% * * *$ & Fator de resistência \\
\hline "Suscetível" $F_{7}$ & \multirow{3}{*}{ Flumetrina } & $3,82(0,24)$ & 0,0008 & $0,0006-0,0010$ & \\
\hline "Santa Luiza" $F_{1}$ & & $2,67(0,15)$ & 0,1235 & $0,0520-0,1972$ & 147,56 \\
\hline "Santa Luiza" $F_{7}$ & & $2,93(0,36)$ & 0,0471 & $0,0472-0,0522$ & 56,29 \\
\hline "Suscetível" $\mathrm{F}_{7}$ & \multirow{3}{*}{ Deltametrina } & $2,11(0,19)$ & 0,0097 & $0,0049-0,0150$ & \\
\hline "Santa Luiza" $F_{1}$ & & $4,11(0,26)$ & 0,6865 & $0,5135-0,9600$ & 70,61 \\
\hline "Santa Luiza" $F_{7}$ & & $4,12(0,39)$ & 0,3813 & $0,3464-0,4298$ & 39,21 \\
\hline "Suscetível" $\mathrm{F}_{7}$ & \multirow{3}{*}{ Cipermetrina } & $2,94(0,20)$ & 0,0478 & $0,0353-0,0641$ & \\
\hline "Santa Luiza" $F_{1}$ & & $3,64(0,20)$ & 1,9966 & $1,7530-2,2773$ & 41,70 \\
\hline "Santa Luiza" $F_{7}$ & & $2,85(0,25)$ & 1,1077 & $0,6082-2,4182$ & 23,3 \\
\hline
\end{tabular}

*Grau técnico.

** $\mathrm{CL}_{50}$ Concentração letal 50.

*** Intervalo de Confiança a 95\%.

laboratório de cepas de carrapatos com uma fração heterozigótica acima de $30 \%$, sem estar em contacto com estes acaricidas, favorece o incremento da fração heterozigótica, diminuindo o nível de resistência em direção a esta família de ixodicidas. Este comportamento é contrário ao obtido com a cepa "Mora" resistente a organofosforados e piretróides, a qual, pelo nível de homozigose de resistência próximo a $90 \%$, não tem permitido a recombinação dos heterozigotos, razão pela qual não se tenha observado uma reversão da resistência frente aos piretróides nesta cepa, mesmo após sete anos de sua manutenção em laboratório. Ao nível do campo, tem-se observado, em populações de carrapatos B. microplus tipo "Mora", resistentes a organofosforados e piretróides, que, ao se deixar de pressionar com piretróides por mais de sete anos, houve uma recuperação da efetividade nos produtos da família dos piretróides, o que sugere a possível recombinação com indivíduos suscetíveis externos à população (SANTA MARIA et al., 1999).

A cepa "Cavalcanti", no Brasil, obteve um FR de 66,6 com flumetrina, a qual apresenta uma resposta toxicológica similar à "Santa Luiza" na geração $F_{7}$ com um FR de 56,29. Estudos de laboratório com carrapatos adultos de cepas brasileiras apresentaram percentuais de controle entre 40,2 e 100\% com o amitraz, enquanto que o obtido com a cepa "Santa Luiza", no México, foi de 58,41\%, o que supõe a existência de diferentes níveis de resistência no estado do Rio Grande do Sul, Brasil (MARTINS et al., 1995).

Outros bioensaios de laboratório realizados na Austrália com carrapatos adultos, avaliando a eficácia do amitraz frente a cepa "Ulam”, indicaram um percentual de controle de $69,1 \%$. Entretanto, devido à característica de resistência semidominante, detectada nas cepas australianas resistentes ao amitraz, realizaram-se estudos combinando carrapatos com características de resistência heterozigótica ao amitraz com carrapatos homozigotos susceptíveis, obtendose somente um incremento de 2 a $3 \%$ de controle nos híbridos resultantes ao utilizar-se este produto no dobro da concentração comercial recomendada. A cepa "Santa Luiza" obteve 58,41\% de controle com o amitraz, registro similar ao observado na Austrália, sugerindo que aumentar ou duplicar a concentração comercial recomendada desse carrapaticida no campo não resolveria o problema do controle neste tipo de cepa (KEMP et al., 1999).

Com referência aos bioensaios utilizandose teleóginas frente aos piretróides, a média de controle foi de 1,5\% para cipermetrina e flumetrina com a cepa brasileira "Cavalcanti", percentual maior ao obtido no México com a cepa "Santa Luiza" para cipermetrina $(1,04 \%)$ e menor ao obtido com a flumetrina de $83,69 \%$ de controle (MARTINS et al., 1995). Para os organofosforados, a cepa "Cavalcanti" mostrou um percentual de controle de $50,42 \%$ com o coumafós, maior ao registrado no México com a cepa "Santa Luiza" que foi de 21,44\% (FURLONG, 1999).

De acordo com os resultados obtidos com a Técnica de Imersão Larval, a cepa "Santa Luiza" da geração $\mathrm{F}_{1}$ apresentou $30 \%$ de heterozigose frente ao amitraz e um FR de 3,76. Posteriormente, com a geração $\mathrm{F}_{7}$, realizou-se uma pressão química com o amitraz, para diminuir a fração heterozigótica e aumentar o grau de homozigose de resistência, observando-se uma diminuição de $10 \%$ na heterozigose e um aumento no fator de resistência que foi de 21,57. A seleção com 
Tabela 5 - Determinação da CL 50 e fator de resistência da cepa "Santa Luiza" de B. microplus com Amitraz CE* através do teste de imersão de larvas.

\begin{tabular}{|c|c|c|c|c|}
\hline Cера & "Slope" ( Erro Padrão) & $\mathrm{CL}_{50} * *$ & IC $95 \% * * *$ & Fator de Resistência \\
\hline "Suscetível" $\mathrm{F}_{7}$ & $2,24(0,08)$ & 0,00001 & $0,00001-0,00002$ & \\
\hline "Santa Luiza" $F_{1}$ & $1,47(0,095)$ & 0,00004 & $0,00002-0,00006$ & 3,76 \\
\hline "Santa Luiza" $F_{7}$ & $2,37(0,10)$ & 0,00023 & $0,00019-0,00026$ & 21,57 \\
\hline
\end{tabular}

*Concentrado Emulsionável.

$* * \mathrm{CL}_{50}$ Concentração letal 50 .

$* * * \mathrm{IC}_{95}$ Intervalo de confiança a $95 \%$.

uma única pressão química de amitraz na cepa "Santa Luiza", em laboratório, ocasionou que o nível de resistência considerado leve antes da pressão química incrementou-se a uma resistência moderada (STONE, 1972).

O processo de purificação da cepa "Santa Luiza" permitiu a validação de um método de diagnóstico de resistência ao amitraz no México, mediante a técnica de imersão larval. Os ensaios de dose-mortalidade, utilizando-se o teste do pacote de larvas preconizado pela FAO, têm proporcionado resultados menos satisfatórios em conseqüência das respostas nãolineares comumente observadas (KEMP et al., 1999). O monitoramento das populações de carrapatos, com relação aos carrapaticidas em uso através de testes in vitro, é um procedimento essencial na detecção precoce de problemas de resistência. Os resultados da caracterização toxicológica desta cepa podem ser utilizados como referencial para a futura identificação de populações de carrapatos resistentes.

\section{REFERÊNCIAS BIBLIOGRÁFICAS}

DRUMMOND, O.R. et al. Tests of acaricides for control of Boophilus annulatus and B. microplus. J Econ Entomol, v.69, p.37-40, 1976.

FURLONG, J. Diagnosis of the susceptibility of the cattle tick, Boophilus spp to acaricides in Minas Gerais State, Brazil. In: SEMINARIO INTERNACIONAL DE PARASITOLOGIA ANIMAL, 4., 1999, Puerto Vallarta, México. Proceedings... Puerto Vallarta : CONASAGA, 1999, p.41-46.

KEMP, H.D. et al. Acaricide resistance in the cattle-ticks Boophilus microplus and $\boldsymbol{B}$. decoloratus: review of resistance data; standardisation of resistance tests and reccomendations for integrated parasite control to delay resistance. Report to the animal health services, AGAH. Rome, Italy : FAO, 1998. $37 \mathrm{p}$.

KEMP, H.D. et al. Strategies for tick control in a world of acaricide resistance. In: SEMINARIO INTERNACIONAL DE PARASITOLOGIA ANIMAL, 4., 1999, Puerto Vallarta, México. Proceedings... CONASAG, 1999. p.1-10.

KUNZ, E.S.; KEMP, H.D. Insecticides and acaricides: Resistance and environmental impact. Rev Sci Tech Off Int Epiz, v.13, n.4, p.1249-1286, 1994.
LEORA SOFTWARE. Polo-PC:A user's guide to probit or logit analysis. Berkeley, CA, USA : LeOra Software, 1987. 22p.

MARTINS, J.R. et al. A situation report on resistance to acaricides by the cattle tick Boophilus microplus in the state of Rio Grande do Sul, Southern Brazil. In: SEMINARIO INTERNACIONAL DE PARASITOLOGIA ANIMAL, "Resistencia y control en garrapatas y moscas de importancia veterinaria", 3., 1995, Acapulco, México. Proceedings... Acapulco : INIFAP, 1995. p.1-8.

NOLAN, J. Current developments in resistance to amidine and pyrethroid tickicides in Australia. In: WHITEHEAD,G.B.; GIBSON, J.D. Tick biology and control. Rhodes University: Grahamstown, 1981. p.109-114.

ORTIZ, E.M. et al. Caracterización de la resistencia de $\boldsymbol{B}$. microplus a ixodicidas en México. In: SEMINARIO INTERNACIONAL DE PARASITOLOGIA ANIMAL, 3., 1995, Acapulco, México. Proceedings... Acapulco : INIFAP, 1995. p.45-57.

ROBERTSON, L.J.; PREISLER, K.H. Pesticide bioassays with arthropods. Boca Raton, FL, USA : CRC, 1992. Cap.3, p. 17-34.

SANTAMARIA, V.M. et al. Análisis de la situación actual mediante el monitoreo de susceptibilidad a ixodicidas en Boophilus microplus de 1993 a 1999 y medidas preventivas para retardar la resistencia al amitraz en México. In: SEMINARIO INTERNACIONAL DE PARASITOLOGIA ANIMAL, 4., 1999, Puerto Vallarta, México. Proceedings... Puerto Vallarta : CONASAG, 1999. p.103117.

SHAW, R.D. Culture of an organophosphorus resistant strain of B. microplus (Can.) and an assessment of its resistance spectrum. Bull Entomol Res v.56, p.389-405, 1966.

STONE, B.F.; HAYDOCK, K.P. A method for measuring the acaricide susceptibility of the cattle B.microplus (Can.). Bull Entomol Res, v.53, p.563-578, 1962.

STONE, B.F. The genetics of resistance by ticks to acaricides. Aust Vet J, v.48, p.345-350, 1972.

STRYDOM, T.; PETER, R. Acaricides and Boophilus spp resistance in South Africa. In: SEMINARIO INTERNACIONAL DE PARASITOLOGIA ANIMAL, 4., 1999, Puerto Vallarta, México. "Control de la Resistencia en Garrapatas, Moscas de Importancia Veterinaria y Enfermedades que Transmiten". Proceedings... Puerto Vallarta : CONASAG, 1999, p.35-40. 